\title{
Bidirectional Phrase-based Statistical Machine Translation
}

\author{
Andrew Finch \\ NICT, Keihanna Science City, \\ Kyoto, 619-0288, Japan \\ andrew.finch@nict.go.jp
}

\author{
Eiichiro Sumita \\ NICT, Keihanna Science City, \\ Kyoto, 619-0288, Japan \\ eichiro.sumita@nict.go.jp
}

\begin{abstract}
This paper investigates the effect of direction in phrase-based statistial machine translation decoding. We compare a typical phrase-based machine translation decoder using a left-to-right decoding strategy to a right-to-left decoder. We also investigate the effectiveness of a bidirectional decoding strategy that integrates both mono-directional approaches, with the aim of reducing the effects due to language specificity. Our experimental evaluation was extensive, based on 272 different language pairs, and gave the surprising result that for most of the language pairs, it was better decode from right-to-left than from left-to-right. As expected the relative performance of left-to-right and rightto-left strategies proved to be highly language dependent. The bidirectional approach outperformed the both the left-toright strategy and the right-to-left strategy, showing consistent improvements that appeared to be unrelated to the specific languages used for translation. Bidirectional decoding gave rise to an improvement in performance over a left-to-right decoding strategy in terms of the BLEU score in $99 \%$ of our experiments.
\end{abstract}

\section{Introduction}

Human language production by its very nature is an ordered process. That is to say, words are written/uttered in a sequence. The current generation of phrase-based statistical machine translation (SMT) systems also generate their target word sequences according to an order. Since the generation process is symmetrical, there are two possible strategies that could be used to generate the target: from beginning to end; or from end to be- ginning. Generating the target in the 'wrong' direction (the opposite direction to the way in which humans do) is counter intuitive, and possibly as a result of this, SMT systems typically generate the target word sequence in the same order as human language production. However it is not necessarily the case that this is most effective strategy for all language pairs. In this paper we investigate the effect of direction in phrase-based SMT decoding.

For the purposes of this paper, we will refer to target word sequence generation that follows the same order as human language production as forward generation, and generation in the opposite direction to human language production as reverse generation. These are often referred "left-toright" and "right-to-left" respectively in the literature, but we avoid this notation as many languages are naturally written from right-to-left.

In earlier work (Watanabe and Sumita, 2002), it was hypothesized that the optimal direction for decoding was dependent on the characteristics of the target language. Their results show that for Japanese to English translation a reverse decoding strategy was the most effective, whereas for English to Japanese translation, a forward decoding strategy proved superior. In addition they implemented a bidirectional decoder, but their results were mixed. For English to Japanese translation, decoding bidirectionally gives higher performance, but for Japanese to English translation they were unable to improve performance by decoding bidirectionally. Their experiments were performed using a decoder based on IBM Model 4 using the translation techniques developed at IBM (Brown et al., 1993).

This work is closely related to the techniques proposed in (Watanabe and Sumita, 2002), but in our case we decode within the framework of a phrase-based SMT system, rather than the IBM model. Our intention was to explore the effect of direction in decoding within the context of a more 
contemporary machine translation paradigm, and to experiment with a broader range of languages. The underlying motivation for our studies however remains the same. Languages have considerably different structure, and certain grammatical constructs tend to occupy particular positions within sentences of the same language, but different positions across languages. These differences may make it easier to tackle the automatic translation of a sentence in a given language from a particular direction. Our approach differs in that the decoding process of a phrased-based decoder is quite different from that used by (Watanabe and Sumita, 2002) since decoding is done using larger units making the re-ordering process much simpler. In (Watanabe and Sumita, 2002) only one language pair is considered, for our experiments we extended this to include translation among 17 different languages including the Japanese and English pair used in (Watanabe and Sumita, 2002). We felt that it was important to consider as many languages as possible in this study, as intuition and evidence from the original study suggests that the effect of direction in decoding is likely to be strongly language dependent.

The next section briefly describes the mechanisms underlying phrase-based decoding. Then we explain the principles behind the forward, reverse and bidirectional decoding strategies used in our experiments. Section 3 presents the experiments we performed. Section 4 gives the results and some analysis. Finally in Section 5, we conclude and offer possible directions for future research.

\section{Phrase-based Translation}

For our experiments we use the phrase-based machine translation techniques described in (Koehn, 2004) and (Koehn et al., 2007), integrating our models within a log-linear framework (Och and Ney, 2002).

One of the advantages of a log-linear model is that it is possible to integrate a diverse set of features into the model. For the decoders used in the experiments in this paper, we included the following feature functions:

- An $n$-gram language model over the target word sequence

- Ensures the target word sequence is a likely sequence of words in the target language
- A phrase translation model

- Effects the segmentation of the source word sequence, and is also responsible for the transformation of source phrases into target phrases.

- A target word sequence length model

- Controls the length of the target word sequence. This is usually a constant term added for each word in the translation hypothesis.

\section{- A lexicalized distortion model}

- Influences the reordering of the translated source phrases in the target word sequence using lexical context on the boundaries of the phrases being reordered.

\subsection{Decoding}

In a phrase-based SMT decoder, the word sequence of the target language is typically generated in order in a forward manner. The words at the start of the translation are generated first, then the subsequent words, in order until the final word of the target word sequence is generated. As the process is phrase-based, the translation is generated in a phrase-by-phrase manner, rather word-by-word. The basic idea is to segment the source word sequence into subsequences (phrases), then translate each phrase individually, and finally compose the target word sequence by reordering the translations of the source phrases. This composition must occur in a particular order, such that target words are generated sequentially from the start (or end in the case of reverse decoding) of the sentence. The reason that the target needs to be generated sequentially is to allow an $n$-gram language model to be applied to the partial target word sequence at each step of the decoding process.

This process is illustrated in Figure 1. In the decoding for both forward and reverse decoders the source sentence is segmented into 2 phrases: "where is" and "the station" (although in this example the segmentation is the same for both decoding strategies, it is not necessarily the case since the search processes are different). In the forward decoding process, first the English phrase "the station" is translated into the Japanese phrase "eki wa". Initially the target sequence consists 


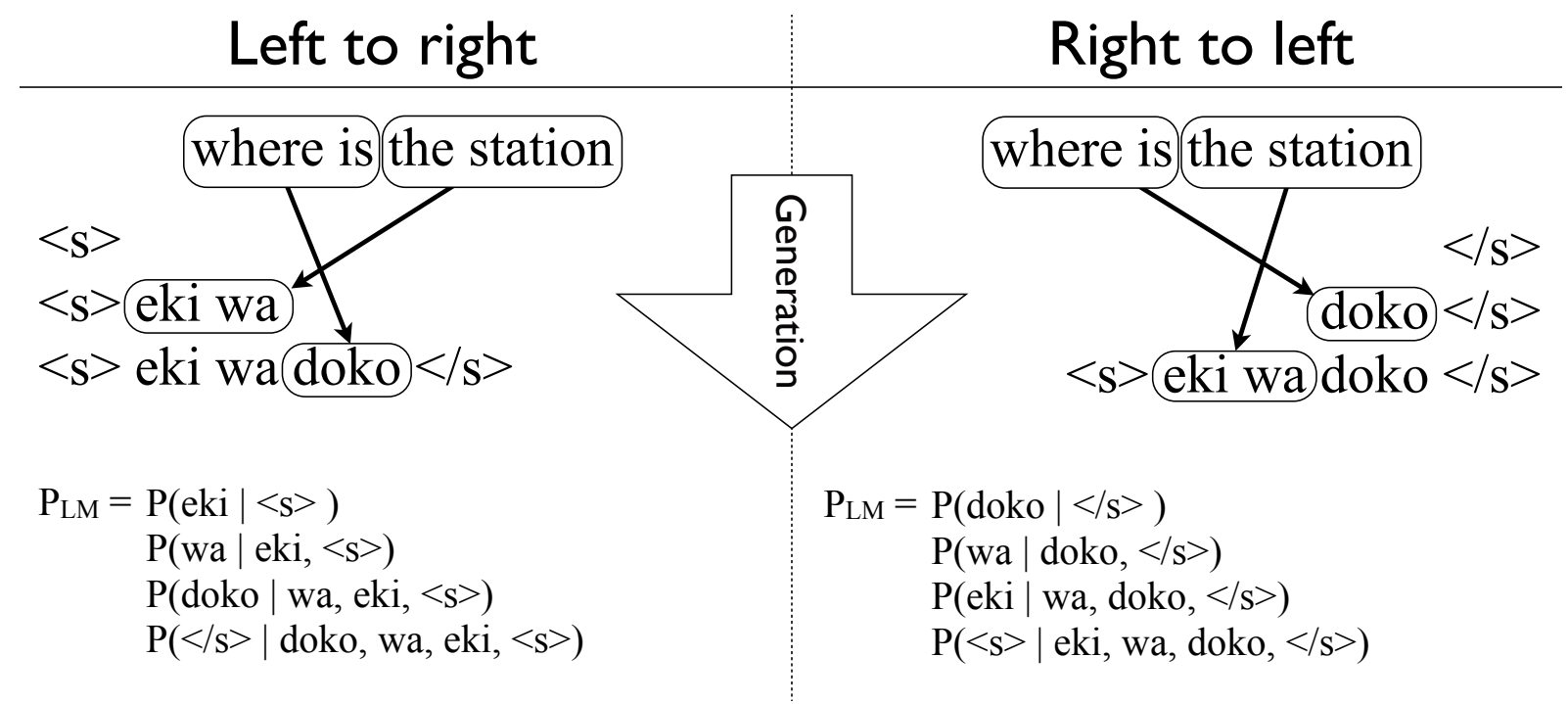

Figure 1: The phrase-based decoding process for an English to Japanese translation, in both forward and reverse directions. The $n$-gram language model probability calculation for the completed translation hypotheses are also shown on the bottom of the figure. See Section 2.1 for a description of the decoding process.

of only the start of sentence marker " $\langle\mathrm{s}\rangle$ ". This marker only serves as context to indicate the start of the sequence for the benefit of the language model. The first target phrase is separated into its component words and each word is added in order to the target word sequence. Each addition causes an application of the language model, hence in Figure 1 the first term of $P_{L M}$ is $P($ eki $\mid\langle\mathrm{s}\rangle)$, the second is $P(\mathrm{wa} \mid\langle\mathrm{s}\rangle)$ and so on. For reverse decoding, the target sentence is generated starting from the end of sentence marker $\langle/ s\rangle$ with the language model context being to the right of the current word. For the case of bidirectional decoding, the model probability for the hypothesis is a linear interpolation of the scores for both forward and reverse hypotheses.

\subsection{Direction in Decoding}

Direction in decoding influences both the models used by the decoder and the search process itself. The direction of decoding determines the order in which target words are generated, the source phrases being translated in any order, therefore it is likely to be features of the target language rather than those of the the source language that determine the effect that the decoding direction has on decoder performance.

\subsubsection{The Language Model}

The fundamental difference between the language models of a forward decoder and that of a reverse decoder is the direction in which the model looks for its context. The forward model looks back to the start of the sentence, whereas the reverse model looks forward to the end of the sentence.

\subsubsection{The Search}

Assuming a full search, a unigram language model and no limitations on reordering, the forward and reverse decoding processes are equivalent. When these constraints are lifted, as is the case in the experiments in this paper, the two search processes diverge and can give rise to hypotheses that are different in character.

The partial hypotheses from early in the search process for forward decoding represent hypotheses for the first few words of the target word sequence, whereas the early partial hypotheses of a reverse decoder hold the last few words. This has two consequences for the search. The first is that (assuming a beam search as used in our experiments), certain candidate word sequences in the early stages of the search might be outside the beam and be pruned. The consequence of this is that sentences that start with (or end with in the case of reverse decoding) the pruned word sequence will not be considered during the remainder of the search. The second is that word se- 
quences in the partial hypotheses are used in the context of the models used in the subsequent decoding. Thus, correctly decoding the start (or end for reverse decoding) of the sentence will benefit the subsequent decoding process.

\section{Experiments}

\subsection{Experimental Data}

The experiments were conducted on all possible pairings among 17 languages. A key to the acronyms used for languages together with information about their respective characteristics is given in Table 1.

We used all of the first ATR Basic Travel Expression Corpus (BTEC1) (Kikui et al., 2003) for these experiments. This corpus contains the kind of expressions that one might expect to find in a phrase-book for travelers. The corpus is similar in character to the IWSLT06 Evaluation Campaign on Spoken Language Translation (Paul, 2006) J-E open track. The sentences are relatively short (see Table 1) with a simple structure and a fairly narrow range of vocabulary due to the limited domain.

The experiments were conducted on data that contained no case information, and also no punctuation (this was an arbitrary decision that we believe had no impact on the results).

We used a 1000 sentence development corpus for all experiments, and the corpus used for evaluation consisted of 5000 sentences with a single reference for each sentence.

\subsection{Training}

Each instance of the decoder is a standard phrasebased machine translation decoder that operates according to the same principles as the publicly available PHARAOH (Koehn, 2004) and MOSES (Koehn et al., 2007) SMT decoders. In these experiments 5-gram language models built with Witten-Bell smoothing were used along with a lexicalized distortion model. The system was trained in a standard manner, using a minimum error-rate training (MERT) procedure (Och, 2003) with respect to the BLEU score (Papineni et al., 2001) on held-out development data to optimize the loglinear model weights. For simplicity, the MERT procedure was performed on independently on the forward and reverse decoders for the bidirectional system, rather them attempting to tune the parameters for the full system.

\subsection{Translation Engines}

\subsubsection{Forward}

The forward decoding translation systems used in these experiments represent the baseline of our experiments. They consist of phrase-based, multistack, beam search decoders commonly used in the field.

\subsubsection{Reverse}

The reverse decoding translation systems used in these experiments were exactly the same as the forward decoding systems. The difference being the that word sequences in the training, development, and source side of the test corpora were reversed prior to training the systems. The final output of the reverse decoders was reordered in a post processing step before evaluation.

\subsubsection{Bidirectional}

The decoder used for the bidirectional decoding experiments was modified in order to be able to decode both forward and reverse in separate instances of the decoder. Models for decoding in forward and reverse directions are loaded, and two decoding instances created. Scores for hypotheses that share the same target word sequence from the two decoders were combined at the end of the decoding process linearly using equal interpolation weights. Hypotheses that were generated by only one of the component decoders were not pruned. The scores from these hypotheses only had a contribution from the decoder that was able to generate them, the contribution from the other decoder being zero.

\subsection{Decoding Constraints}

The experiments reported in this paper were conducted with loose constraints on the decoding as overconstraining the decoding process could lead to differences between unidirectional and bidirectional strategies. More specificially, the decoding was done with a beam width of 100 , no beam thresholding and no constraints on the reordering process. Figure 2 shows the effect of varying the beam width (stack size) in the search for forward decoder of the English to Japanese translation experiment. At the beam width of 100 used in our experiments, the gains from doubling the beam with are small (0.07 BLEU percentage points).

It is also important to note that a future cost identical to that used in the MOSES decoder 


\begin{tabular}{|c||l|r|c|r|l|}
\hline Abbreviation & Language & \#Words & Avg. sent length & Vocabulary & Order \\
\hline \hline ar & Arabic & 806853 & 5.16 & 47093 & SVO \\
\hline da & Danish & 806853 & 5.16 & 47093 & SVO \\
\hline de & German & 907354 & 5.80 & 23443 & SVO \\
\hline en & English & 970252 & 6.21 & 12900 & SVO \\
\hline es & Spanish & 881709 & 5.64 & 18128 & SVO \\
\hline fr & French & 983402 & 6.29 & 17311 & SVO \\
\hline id & Indonesian (Malay) & 865572 & 5.54 & 15527 & SVO \\
\hline it & Italian & 865572 & 5.54 & 15527 & SVO \\
\hline ja & Japanese & 1149065 & 7.35 & 15405 & SOV \\
\hline ko & Korean & 1091874 & 6.98 & 17015 & SOV \\
\hline ms & Malaysian (Malay) & 873959 & 5.59 & 16182 & SVO \\
\hline nl & Dutch & 927861 & 5.94 & 19775 & SVO \\
\hline pt & Portuguese & 881428 & 5.64 & 18217 & SVO \\
\hline ru & Russian & 781848 & 5.00 & 32199 & SVO \\
\hline th & Thai & 1211690 & 7.75 & 6921 & SVO \\
\hline vi & Vietnamese & 1223341 & 7.83 & 8055 & SVO \\
\hline zh & Chinese & 873375 & 5.59 & 14854 & SVO \\
\hline
\end{tabular}

Table 1: Key to the languages, corpus statistics and word order. SVO denotes a language that predominantly has subject-verb-object order, and SOV denotes a language that predominantly has subject-objectverb order

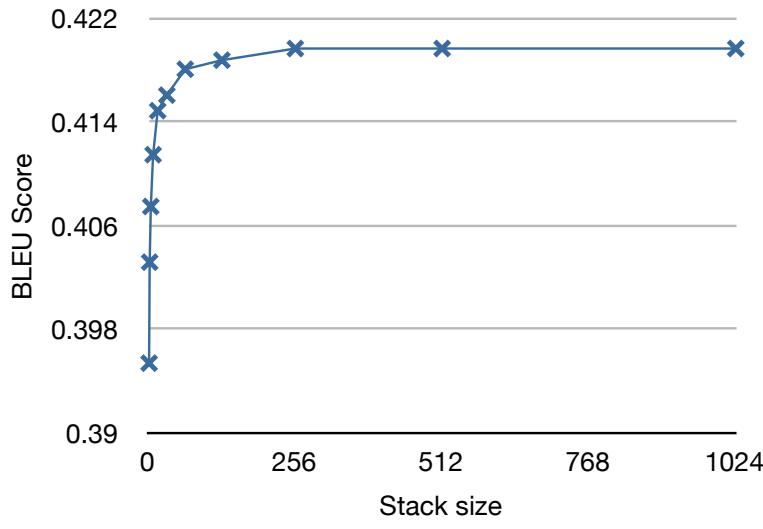

Figure 2: The performance of a forward decoder (En-Ja) with increasing stack size.

(Koehn et al., 2007) was also included in the scores for partial hypothesis during the decoding.

\subsection{Computational Overhead}

In the current implementation, bidirectional decoding takes twice as long as a mono-directional system. However, in a multi-threaded environment, each instance of the decoder is able to run on its own thread in parallel, and so this slowdown can be mitigated in some circumstances. Future generations of the bidirectional decoder will more tightly couple the two decoders, and we believe this will lead to faster and more effective search.

\subsection{Evaluation}

The results presented in this paper are given in terms of the BLEU score (Papineni et al., 2001). This metric measures the geometric mean of $n$ gram precision of $n$-grams drawn from the output translation and a set of reference translations for that translation.

There are large number of proposed methods for carrying out machine translation evaluation. Methods differ in their focus of characteristics of the translation (for example fluency or adequacy), and moreover anomolous results can occur if a single metric is relied on. Therefore, we also carried out evaluations using the NIST (Doddington, 2002), METEOR (Banerjee and Lavie, 2005), WER (Hunt, 1989), PER (Tillmann et al., 1997) and TER (Snover et al., 2005) machine translation evaluation techniques.

\section{Results}

The results of the experiments in terms of the BLEU score are given in Tables ??, 5, 3 and 3 . These results show the performance of the reverse and bidirectional decoding strategies relative to the usual forward decoding strategy. The cells in the tables that represent experiments in which 


\begin{tabular}{|c||r|r|r|r|r|r|r|r|r|r|r|r|r|r|r|r|r|}
\hline & ar & da & de & en & es & fr & id & it & ja & ko & ms & nl & pt & ru & th & vi & zh \\
\hline \hline ar & - & 47.8 & 48.8 & 51.7 & 48.8 & 47.3 & 46.5 & 49.2 & 29.8 & 27.8 & 46.9 & 49.0 & 49.0 & 47.8 & 39.7 & 43.0 & 27.8 \\
\hline da & 58.3 & - & 58.7 & 63.0 & 58.6 & 55.7 & 53.5 & 58.5 & 37.5 & 35.1 & 54.4 & 59.6 & 59.0 & 55.4 & 48.1 & 51.7 & 35.2 \\
\hline de & 53.8 & 55.5 & - & 59.4 & 55.9 & 51.9 & 50.3 & 55.3 & 34.2 & 32.0 & 50.8 & 57.0 & 55.9 & 51.2 & 45.7 & 48.9 & 32.7 \\
\hline en & 63.6 & 65.8 & 64.8 & - & 67.0 & 61.0 & 58.4 & 65.8 & 41.1 & 38.7 & 59.1 & 67.6 & 66.7 & 58.7 & 52.8 & 57.7 & 38.6 \\
\hline es & 57.6 & 58.2 & 58.0 & 65.6 & - & 56.6 & 54.2 & 61.1 & 38.3 & 36.4 & 54.3 & 59.6 & 62.6 & 55.1 & 47.6 & 51.3 & 36.0 \\
\hline fr & 57.8 & 58.3 & 58.0 & 62.3 & 58.9 & - & 52.7 & 57.4 & 39.1 & 37.7 & 53.8 & 58.3 & 57.9 & 54.8 & 47.7 & 50.4 & 37.6 \\
\hline id & 54.7 & 52.8 & 52.8 & 56.6 & 53.7 & 51.0 & - & 53.1 & 37.2 & 35.6 & 86.4 & 53.8 & 53.0 & 51.3 & 46.4 & 48.4 & 34.9 \\
\hline it & 54.1 & 53.4 & 54.4 & 59.4 & 56.4 & 51.8 & 49.2 & - & 34.4 & 32.8 & 49.9 & 55.1 & 56.2 & 50.5 & 44.0 & 47.0 & 33.6 \\
\hline ja & 38.2 & 39.2 & 38.6 & 41.9 & 39.9 & 40.2 & 40.7 & 39.5 & - & 69.4 & 40.4 & 39.5 & 39.7 & 37.8 & 37.3 & 37.2 & 52.1 \\
\hline ko & 34.4 & 35.3 & 34.6 & 38.2 & 36.3 & 36.2 & 36.8 & 35.6 & 66.4 & - & 36.6 & 35.6 & 36.3 & 34.5 & 34.2 & 34.1 & 46.4 \\
\hline ms & 54.5 & 52.7 & 52.6 & 56.2 & 53.4 & 50.6 & 82.5 & 53.2 & 36.8 & 34.9 & - & 53.6 & 53.4 & 51.3 & 46.7 & 49.2 & 34.8 \\
\hline nl & 55.1 & 57.3 & 58.8 & 63.2 & 58.5 & 54.5 & 52.4 & 57.1 & 36.7 & 34.1 & 53.4 & - & 58.3 & 53.5 & 48.7 & 50.7 & 35.2 \\
\hline pt & 56.8 & 57.7 & 57.6 & 63.8 & 62.0 & 55.5 & 52.7 & 59.7 & 37.8 & 36.4 & 53.4 & 58.7 & - & 54.2 & 47.1 & 50.6 & 35.8 \\
\hline ru & 51.4 & 49.1 & 50.2 & 53.3 & 52.0 & 48.7 & 48.6 & 51.6 & 31.9 & 29.5 & 49.1 & 50.9 & 50.5 & - & 41.8 & 43.7 & 30.0 \\
\hline th & 53.8 & 55.0 & 54.8 & 58.2 & 55.8 & 53.3 & 55.0 & 54.8 & 41.4 & 39.2 & 55.4 & 55.9 & 55.5 & 53.0 & - & 56.0 & 40.4 \\
\hline vi & 53.6 & 53.6 & 54.2 & 57.4 & 54.2 & 51.4 & 52.3 & 53.3 & 37.6 & 35.8 & 53.3 & 54.6 & 54.4 & 51.7 & 50.3 & & 36.2 \\
\hline zh & 32.0 & 33.0 & 32.6 & 34.6 & 33.2 & 33.7 & 34.2 & 33.2 & 47.8 & 43.5 & 33.9 & 33.4 & 32.6 & 32.2 & 31.1 & 29.7 & - \\
\hline
\end{tabular}

Table 2: Baseline BLEU scores for all systems. The figures represent the scores in BLEU percentage points of the baseline left-to-right decoding systems. Source languages are indicated by the column headers, the row headers denoting the target languages.

the forward strategy outperformed the contrasting strategy are shaded in gray. The numbers in the cells represent the difference in BLEU percentage points for the systems being compared in that cell.

It is clear from Table 3 that for most of the language pairs $(67 \%$ of them for BLEU, and a similar percentage for all the other metrics except METEOR), better evaluation scores were achieved by using a reverse decoding strategy than a forward strategy. This is a surprising result because language is produced naturally in a forward manner (by definition), and therefore one might expect this to also be the optimal direction for word sequence generation in decoding.

\subsection{Word Order Typography}

Following (Watanabe and Sumita, 2002), to explain the effects we observe in our results we look to the word order typography of the target language (Comrie and Vogel, 2000). The word order of a language is defined in terms of the order in which you would expect to encounter the finite verb (V) and its arguments, subject (S) and object $(\mathrm{O})$. In most languages $\mathrm{S}$ precedes $\mathrm{O}$ and $\mathrm{V}$. Whether or not $\mathrm{O}$ precedes or follows $\mathrm{V}$ defines the two most prevalent word order types SOV and SVO (Comrie and Vogel, 2000).

Two of the target languages in this study
(Japanese and Korean) have the SOV word type, the remainder having the SVO word order type. In Table 3 looking at the rows for $j a$ and $k o$ we can see that for both of these languages reverse decoding outperformed forward decoding in only 4 out of 12 experiments. Furthermore these two languages were the two languages that benefited the most (in terms of the number of experimental cases) from forward decoding. The two languages also agree on the best decoding direction for 12 of the 16 language pairs. This apparent correlation may reflect similarities between the two languages (word order type, or other common features of the languages).

Given this evidence, it seems plausible that word order does account in part for the differences in performance when decoding in differing directions, but this can only be part of the explanation since there are 4 source languages for which reverse decoding yielded higher performance.

It should be noted that our results differ from those of (Watanabe and Sumita, 2002) for English to Japanese translation, who observed gains when decoding in the reverse direction for this language pair. It is hard to compare our results directly with theirs however, due to the differences in the decoders used in the experiments (ours being phrase-based, and theirs based on the IBM ap- 


\begin{tabular}{|c||r|r|r|r|r|r|r|r|r|r|r|r|r|r|r|r|r|}
\hline & ar & da & de & en & es & fr & id & it & ja & ko & ms & nl & pt & ru & th & vi & zh \\
\hline \hline ar & - & 0.87 & 0.34 & 1.30 & 0.93 & 1.63 & 0.66 & 0.58 & 0.12 & 0.36 & 0.85 & 0.33 & 0.88 & 0.22 & 1.33 & 1.04 & 0.88 \\
\hline da & 0.25 & - & 0.41 & 0.71 & 0.56 & 0.70 & 1.10 & 0.31 & 0.46 & 0.07 & 0.96 & 0.13 & 0.62 & 0.17 & 1.28 & 0.71 & 0.29 \\
\hline de & 0.41 & 0.04 & - & 0.38 & 0.52 & 0.15 & 0.80 & 0.01 & 0.47 & 0.72 & 0.60 & 0.25 & 0.21 & 0.05 & 0.47 & 0.68 & 0.20 \\
\hline en & 0.04 & 0.05 & 0.21 & - & 0.05 & 0.13 & 0.58 & 0.02 & 0.73 & 0.35 & 0.39 & 0.07 & 0.52 & 0.05 & 0.67 & 0.63 & 0.29 \\
\hline es & 0.14 & 0.19 & 0.05 & 0.35 & - & 0.68 & 0.01 & 0.08 & 0.25 & 0.31 & 0.25 & 0.25 & 0.17 & 0.07 & 0.43 & 0.44 & 0.78 \\
\hline fr & 0.37 & 0.57 & 0.38 & 0.66 & 0.21 & - & 0.36 & 0.28 & 0.15 & 0.45 & 0.22 & 0.46 & 0.64 & 0.10 & 0.25 & 0.58 & 0.31 \\
\hline id & 0.16 & 0.02 & 0.31 & 1.45 & 0.58 & 0.50 & - & 0.34 & 0.03 & 0.27 & 0.00 & 0.42 & 0.57 & 0.36 & 0.53 & 1.04 & 0.59 \\
\hline it & 0.28 & 0.72 & 0.36 & 0.27 & 0.08 & 0.30 & 0.11 & - & 0.07 & 0.12 & 0.37 & 0.23 & 0.05 & 0.37 & 0.04 & 0.63 & 0.37 \\
\hline ja & 0.36 & 0.22 & 0.03 & 0.03 & 0.22 & 0.13 & 0.64 & 0.36 & - & 0.21 & 0.57 & 0.46 & 0.08 & 0.33 & 0.08 & 0.83 & 0.70 \\
\hline ko & 0.35 & 0.01 & 0.31 & 0.03 & 0.12 & 0.07 & 0.13 & 0.21 & 0.42 & - & 0.29 & 0.07 & 0.42 & 0.40 & 0.44 & 0.62 & 0.05 \\
\hline ms & 0.06 & 0.49 & 0.53 & 1.38 & 0.99 & 0.71 & 0.47 & 0.34 & 0.11 & 0.32 & - & 0.62 & 0.27 & 0.10 & 0.83 & 0.99 & 0.11 \\
\hline nl & 0.26 & 0.03 & 0.26 & 0.30 & 0.20 & 0.19 & 0.47 & 0.23 & 0.13 & 0.06 & 0.06 & - & 0.08 & 0.09 & 0.06 & 1.00 & 0.15 \\
\hline pt & 0.03 & 0.34 & 0.06 & 0.51 & 0.07 & 0.17 & 0.06 & 0.18 & 0.13 & 0.65 & 0.08 & 0.10 & - & 0.06 & 0.09 & 0.85 & 0.35 \\
\hline ru & 0.25 & 0.58 & 0.67 & 0.74 & 0.01 & 0.48 & 0.50 & 0.27 & 0.41 & 0.38 & 0.13 & 0.38 & 0.46 & - & 0.88 & 0.56 & 0.49 \\
\hline th & 0.19 & 0.28 & 0.21 & 0.41 & 0.05 & 0.23 & 0.30 & 0.00 & 0.34 & 0.04 & 0.25 & 0.07 & 0.21 & 0.08 & - & 0.46 & 0.25 \\
\hline vi & 0.21 & 0.34 & 0.24 & 0.65 & 0.72 & 0.34 & 0.06 & 0.59 & 0.24 & 0.22 & 0.19 & 0.12 & 0.11 & 0.18 & 0.63 & - & 0.15 \\
\hline zh & 0.43 & 0.26 & 0.42 & 0.05 & 0.15 & 0.31 & 0.16 & 0.28 & 0.00 & 0.31 & 0.40 & 0.14 & 0.67 & 0.18 & 0.39 & 0.21 & - \\
\hline
\end{tabular}

Table 3: Gains in BLEU score from reverse decoding over a forward decoding strategy The numbers in the cells are the differences in BLEU percentage points between the systems. Shaded cells indicate the cases where forward decoding give a higher score. Source languages are indicated by the column headers, the row headers denoting the target languages.

\begin{tabular}{|l||c|c|c|}
\hline Metric & Bi $>$ For & Bi $>$ Rev & Rev $>$ For \\
\hline \hline BLEU & 98.90 & 84.93 & 67.65 \\
\hline NIST & 98.53 & 78.31 & 75.00 \\
\hline METEOR & 99.63 & 95.96 & 50.74 \\
\hline WER & 99.26 & 92.85 & 66.18 \\
\hline PER & 98.53 & 84.97 & 70.59 \\
\hline TER & 99.63 & 91.18 & 68.75 \\
\hline
\end{tabular}

Table 4: Summary of the results using several automatic metrics for evaluation. Numbers in the table correspond to the percentage of experiments in which the condition at the head of the column was true (for example figure in the first row and first column means that for 98.9 percent of the language pairs the BLEU score for the bidirectional decoder was better than that of the forward decoder)

proach (Brown et al., 1993)).

The results were the similar in character when other MT evaluation methods were used. These results are summarized in Table 3.

\subsection{Bidirectional Decoding}

Table 5 shows the performance of the bidirectional decoder relative to a forward decoder. As can be

seen from the table, in 269 out of the 272 experiments the bidirectional decoder outperformed the unidirectional decoder. The gains ranged from a maximum of 1.81 BLEU (translating from Thai to Arabic) points, to a minimum of -0.04 BLEU points (translating from Indonesian to Japanese) with the average gain over all experiments being 0.56 BLEU points. It is clear from our experiments that there is much to be gained from decoding bidirectionally. Our results were almost unanimously positive, and in all three negative cases the drop in performance was small.

\section{Conclusion}

In this paper we have investigated the effects on phrase-based machine translation performance of three different decoding strategies: forward, reverse and bidirectional. The experiments were conducted on a large set of source and target languages consisting of 272 experiments representing all possible pairings from a set of 17 languages. These languages were very diverse in character and included a broad selection of European and Asian languages. The experimental results revealed that for SVO word order languages it is usually better to decode in a reverse manner, and in contrast, for SOV word order languages it is usu- 


\begin{tabular}{|c||r|r|r|r|r|r|r|r|r|r|r|r|r|r|r|r|r|}
\hline & ar & da & de & en & es & fr & id & it & ja & ko & ms & nl & pt & ru & th & vi & zh \\
\hline \hline ar & - & 0.66 & 0.51 & 1.03 & 0.65 & 0.75 & 0.59 & 0.47 & 0.46 & 0.85 & 0.59 & 0.69 & 0.39 & 0.30 & 1.81 & 1.30 & 0.85 \\
\hline da & 0.27 & - & 0.61 & 0.63 & 0.38 & 0.60 & 0.59 & 0.29 & 1.04 & 0.79 & 0.69 & 0.45 & 0.89 & 0.27 & 1.28 & 0.87 & 0.47 \\
\hline de & 0.52 & 0.51 & - & 0.54 & 0.44 & 0.42 & 0.70 & 0.40 & 0.74 & 0.45 & 0.83 & 0.37 & 0.28 & 0.34 & 0.77 & 0.90 & 0.84 \\
\hline en & 0.53 & 0.01 & 0.32 & - & 0.23 & 0.25 & 0.56 & 0.19 & 1.11 & 0.59 & 0.28 & 0.27 & 0.45 & 0.60 & 0.89 & 0.61 & 0.58 \\
\hline es & 0.28 & 0.48 & 0.45 & 0.56 & - & 0.43 & 0.12 & 0.26 & 0.57 & 0.64 & 0.56 & 0.06 & 0.04 & 0.24 & 1.16 & 1.23 & 0.68 \\
\hline fr & 0.70 & 0.33 & 0.54 & 0.66 & 0.46 & - & 0.49 & 0.57 & 0.24 & 0.13 & 0.11 & 0.43 & 0.33 & 0.55 & 0.91 & 1.09 & 0.57 \\
\hline id & 0.24 & 0.32 & 0.36 & 0.93 & 0.70 & 0.65 & - & 0.35 & 0.75 & 0.77 & 0.11 & 0.46 & 0.69 & 0.57 & 0.99 & 0.85 & 0.47 \\
\hline it & 0.13 & 0.55 & 0.32 & 0.43 & 0.47 & 0.51 & 0.64 & - & 0.65 & 0.42 & 0.77 & 0.51 & 0.51 & 0.69 & 0.85 & 0.98 & 0.58 \\
\hline ja & 0.38 & 0.62 & 0.60 & 0.61 & 0.38 & 0.73 & 0.04 & 0.43 & - & 0.35 & 0.05 & 0.70 & 0.30 & 0.38 & 0.53 & 0.17 & 0.02 \\
\hline ko & 0.49 & 0.62 & 0.90 & 0.40 & 0.34 & 0.57 & 0.47 & 0.47 & 0.02 & - & 0.23 & 0.52 & 0.20 & 0.83 & 0.70 & 0.44 & 0.83 \\
\hline ms & 0.37 & 0.57 & 0.63 & 0.92 & 0.81 & 0.75 & 0.36 & 0.54 & 0.70 & 1.31 & - & 0.76 & 0.35 & 0.51 & 1.14 & 0.70 & 0.35 \\
\hline nl & 0.35 & 0.14 & 0.54 & 0.33 & 0.30 & 0.46 & 0.68 & 0.69 & 0.77 & 0.63 & 0.44 & - & 0.42 & 0.67 & 0.71 & 1.13 & 0.55 \\
\hline pt & 0.46 & 0.21 & 0.37 & 0.21 & 0.17 & 0.49 & 0.47 & 0.24 & 0.88 & 0.45 & 0.54 & 0.39 & - & 0.41 & 0.94 & 1.15 & 0.90 \\
\hline ru & 0.69 & 0.63 & 0.69 & 0.77 & 0.26 & 0.50 & 0.79 & 0.52 & 0.69 & 0.90 & 0.66 & 0.69 & 0.40 & - & 1.19 & 1.23 & 0.47 \\
\hline th & 0.90 & 0.49 & 0.53 & 0.77 & 0.64 & 0.38 & 0.21 & 0.60 & 0.37 & 0.96 & 0.38 & 0.63 & 0.68 & 0.72 & - & 0.33 & 0.45 \\
\hline vi & 0.64 & 0.61 & 0.42 & 1.09 & 0.84 & 0.63 & 0.34 & 0.70 & 0.59 & 0.39 & 0.16 & 0.56 & 0.36 & 0.50 & 0.77 & - & 0.53 \\
\hline zh & 0.23 & 0.48 & 0.96 & 0.33 & 0.49 & 0.32 & 0.27 & 0.43 & 0.43 & 0.69 & 0.31 & 0.97 & 0.85 & 0.23 & 0.40 & 0.50 & - \\
\hline
\end{tabular}

Table 5: Gains in BLEU score from decoding bidirectionally over a forward decoding strategy. The numbers in the cells are the differences in BLEU percentage points between the systems. Shaded cells indicate the cases where forward decoding gave a higher score. Source languages are indicated by the column headers, the row headers denoting the target languages.

ally better to decode in a forward direction. Our main contribution has been to show that a bidirectional decoding strategy is superior to both monodirectional decoding strategies. It might be argued that the gains arise simply from system combination. However, our systems are combined in a simple linear fashion, and gains will only arise when the second system contributes novel and useful information to into the combination. Furthermore, our systems are trained on two copies of the same data, no additional data is required. The gains from decoding bidirectionally were obtained very consistently, with only loose constraints on the decoding. This can be seen clearly in Table 5 where the results are almost unanimously positive. Moreover, these gains appear to be independent of the linguistic characteristics of the source and target languages.

In the future we would like to explore the possibilities created by more tightly coupling the forward and reverse components of the bidirectional decoder. Scores from partial hypotheses of both processes could be combined and used at each step of the decoding, making the search more informed. Furthermore, forward partial hypotheses and reverse hypotheses would 'meet' during decoding (when one decoding direction has covered words in the source that the other has yet to cover), and provide paths for each other to a final state in the search.

\section{Acknowledgment}

This work is partly supported by the Grant-inAid for Scientific Research (C) Number 19500137 and "Construction of speech translation foundation aiming to overcome the barrier between Asian languages", the Special Coordination Funds for Promoting Science and Technology of the Ministry of Education, Culture, Sports, Science and Technology, Japan.

\section{References}

Satanjeev Banerjee and Alon Lavie. 2005. Meteor: an automatic metric for mt evaluation with improved correlation with human judgments. In $A C L$ 2005: Workshop on Intrinsic and Extrinsic Evaluation Measures for Machine Translation and/or Summarization, pages 65-72.

P. Brown, S. Della Pietra, V. Della Pietra, and R.J. Mercer. 1993. The Mathematics of Statistical Machine Translation: Parameter Estimation. Computational Linguistics, 19(2):263-311.

Bernard Comrie and Petra M Vogel, editors. 2000. Approaches to the Typography of Word Classes. Mouton de Gruyter, Berlin. 
G. Doddington. 2002. Automatic Evaluation of Machine Translation Quality Using N-gram CoOccurrence Statistics. In Proceedings of the HLT Conference, San Diego, California.

Melvyn J. Hunt. 1989. Figures of merit for assessing connected-word recognisers. In In Proceedings of the ESCA Tutorial and Research Workshop on Speech Input/Output Assessment and Speech Databases, pages 127-131.

G. Kikui, E. Sumita, T. Takezawa, and S. Yamamoto. 2003. Creating corpora for speech-to-speech translation. In Proceedings of EUROSPEECH-03, pages 381-384.

Philipp Koehn, Hieu Hoang, Alexandra Birch, Chris Callison-Burch, Marcello Federico, Nicola Bertoldi, Brooke Cowa, Wade Shen, Christine Moran, Richard Zens, Chris Dyer, Ondrej Bojar, Alexandra Constantin, and Evan Herbst. 2007. Moses: open source toolkit for statistical machine translation. In ACL 2007: proceedings of demo and poster sessions, pages 177-180, Prague, Czeck Republic, June.

Philipp Koehn. 2004. Pharaoh: a beam search decoder for phrase-based statistical machine translation models. In Machine translation: from real users to research: 6th conference of AMTA, pages 115-124, Washington, DC.

Franz Josef Och and Hermann Ney. 2002. Discriminative training and maximum entropy models for statistical machine translation. In In Proceedings of the 40th Annual Meeting of the Association for Computational Linguistics (ACL 2002), pages 295-302.

Franz J. Och. 2003. Minimum error rate training for statistical machine trainslation. In Proceedings of the ACL.

Kishore Papineni, Salim Roukos, Todd Ward, and WeiJing Zhu. 2001. Bleu: a method for automatic evaluation of machine translation. In $A C L$ '02: Proceedings of the 40th Annual Meeting on Association for Computational Linguistics, pages 311-318, Morristown, NJ, USA. Association for Computational Linguistics.

Michael Paul. 2006. Overview of the iwslt 2006 evaluation campaign. In Proceedings of the IWLST.

Mathew Snover, Bonnie Dorr, Richard Schwartz, John Makhoul, Linnea Micciula, and Ralph Weischedel. 2005. A study of translation error rate with targeted human annotation. Technical report, University of Maryland, College Park and BBN Technologies, July.

C. Tillmann, S. Vogel, H. Ney, A. Zubiaga, and H. Sawaf. 1997. Accelerated dp based search for statistical translation. In In European Conf. on Speech Communication and Technology, pages 2667-2670.
Taro Watanabe and Eiichiro Sumita. 2002. Bidirectional decoding for statistical machine translation. In Proceedings of the 19th international conference on Computational linguistics, pages 1-7, Morristown, NJ, USA. Association for Computational Linguistics. 